\title{
Motor response to levodopa in patients with parkinsonian motor fluctuations: a follow-up study over three years
}

\author{
Andrew J Hughes, Jonathan P Frankel, Peter A Kempster, Gerald M Stern, \\ Andrew J Lees
}

\begin{abstract}
To clarify the way in which the clinical response to levodopa changes with the progression of Parkinson's disease, a longitudinal study was performed to quantify motor response characteristics to single doses of levodopa by mouth over three years in 23 patients with fluctuating motor function. A significant increase in motor disability in "on" (time of peak motor improvement) and "off" (before levodopa dose) phases occurred and "on" phase dyskinesia increased by $24 \%$, though the amplitude of motor response was conserved. There was no evidence of progressive loss of response of certain motor deficits affecting axial muscles and gait. The mean duration of motor response decreased by $17 \%$. Both shortening of response duration and increase in "off" phase disability contribute to the development of motor fluctuations. A short response time to the levodopa test dose was not an invariable finding in patients with severe fluctuations, whereas all had large response amplitudes and high "off" phase disability scores. Patients who have developed motor fluctuations may continue to respond to dopaminergic treatment until late in the disease course, despite the unstable nature of their responses.
\end{abstract}

$(F$ Neurol Neurosurg Psychiatry 1994;57:430-434)

Although levodopa remains the most physiological and probably the best available pharmacological treatment for Parkinson's disease, many different patterns of clinical response are seen. Levodopa usually produces significant initial improvement, though a disappointing lack of benefit occurs in a few patients. After several years of treatment, a stable response often gives way to fluctuating motor function. Some patients with advanced disease manifest wild and unpredictable swings in motor function, whereas others are severely disabled, do not fluctuate, and respond poorly to drug treatment. ${ }^{1}$ Even in an individual patient there may be topographic differences in the magnitude of response to levodopa, or tremor, bradykinesia, and rigidity may respond differentially. The causes of these variations in response are uncertain. It has been argued that a progressive shortening of the duration of response to levodopa is the critical factor for the emergence of motor fluctuations, ${ }^{2}$ whereas others point to increasing disability during phases of lapse in levodopa response and a consequent widening of amplitude of motor fluctuations. ${ }^{3}$ The disability seen late in the course of Parkinson's disease has been considered to be mainly due to the emergence of levodopa-resistant features such as postural instability, cognitive impairment and bulbar dysfunction, rather than to a loss of response of cardinal motor features. ${ }^{4}$ These conclusions have been based largely on cross-sectional studies of patients with different durations of disease and levodopa treatment, assuming a common pattern and rate of disease progression.

On the basis that a longitudinal study is likely to give a more accurate picture of the evolution of response to levodopa which accompanies disease progression, we have measured the magnitude, duration, and pattern of response to single oral doses of levodopa over a three year period in 23 patients.

\section{Patients and methods}

Twenty-three patients (13 men, 10 women) were studied. All fulfilled the United Kingdom Parkinson's disease brain bank criteria $^{5}$ for a diagnosis of idiopathic Parkinson's disease. Their mean (range) age at first assessment was 57 (39-73) years and the mean duration of disease was 12 (4-23) years. All patients were receiving levodopa/ decarboxylase inhibitor drugs and the mean (range) duration of this treatment was 11 (2-19) years. All but one had symptomatic motor fluctuations and levodopa-induced dyskinetic involuntary movements at the start of the study and the remaining patient developed these during the study period. Table 1 summarises the clinical details.

Patients were studied under identical standardised conditions on two occasions separated by a mean (range) period of $37(26-43)$ months. A single dose of levodopa $250 \mathrm{mg} /$ carbidopa $25 \mathrm{mg}$ was given early in the morning after overnight fasting. All drugs had been withheld for at least 10 hours. Patients continued to fast until the conclusion of the assessment of dose response. To ensure standardisation of assessment methods, one of the investigators (JPF) took part in the two studies. Serial motor assessments were carried out at 15 minute intervals until the motor response had worn off, as judged by comparison with the motor function before the drug dose. 
Table 1 Clinical features of all patients including "off" and "on" modified Webster scale scores at time of initial study

\begin{tabular}{|c|c|c|c|c|c|c|}
\hline \multirow[b]{2}{*}{ Patient no } & \multirow{2}{*}{$\begin{array}{l}\text { Age (years) } \\
\text { /Sex }\end{array}$} & \multirow{2}{*}{$\begin{array}{l}\text { Duration } \\
\text { of disease } \\
\text { (years) }\end{array}$} & \multirow{2}{*}{$\begin{array}{l}\text { Duration } \\
\text { of levodopa } \\
\text { treatment (years) }\end{array}$} & \multicolumn{2}{|c|}{ Modified Webster score } & \multirow{2}{*}{$\begin{array}{l}\text { Duration of } \\
\text { motor response to } \\
\text { levodopa (minutes) }\end{array}$} \\
\hline & & & & “Off" & “On" & \\
\hline 1 & $39 / F$ & 4 & 2 & 16 & 2 & 100 \\
\hline 2 & $56 / \mathrm{M}$ & 4 & 3 & 11 & 8 & 255 \\
\hline 3 & $43 / \mathrm{M}$ & 5 & 5 & 20 & 5 & 215 \\
\hline 4 & $56 / \mathrm{M}$ & 5 & 5 & 9 & 3 & 320 \\
\hline 5 & $67 / \mathrm{F}$ & 8 & 7 & 25 & 11 & 165 \\
\hline 6 & 49/M & 8 & 6 & 29 & 12 & 345 \\
\hline 7 & $61 / M$ & 9 & 7 & 12 & 2 & 195 \\
\hline 8 & $63 / \mathrm{F}$ & 10 & 10 & 20 & 3 & 300 \\
\hline 9 & $52 / \mathrm{M}$ & 10 & 10 & 17 & 1 & 225 \\
\hline 10 & $49 / \mathrm{M}$ & 10 & 10 & $\mathrm{NA}^{\star}$ & $\mathrm{NA}^{\star}$ & 225 \\
\hline 11 & $53 / \mathrm{M}$ & 10 & 10 & 25 & 8 & 180 \\
\hline 12 & $73 / \mathrm{M}$ & 12 & 10 & 21 & 7 & 180 \\
\hline 13 & $39 / \mathrm{F}$ & 13 & 11 & 28 & 5 & 135 \\
\hline 14 & $54 / \mathrm{F}$ & 14 & 14 & 24 & 5 & 195 \\
\hline 15 & $66 / \mathrm{F}$ & 14 & 13 & 23 & 6 & 120 \\
\hline 16 & 59/M & 15 & 15 & 14 & 5 & 130 \\
\hline 17 & $63 / \mathrm{F}$ & 15 & 10 & 16 & 8 & 180 \\
\hline 18 & $69 / \mathrm{M}$ & 17 & 12 & 25 & 10 & 260 \\
\hline 19 & $63 / \mathrm{F}$ & 17 & 15 & 26 & 6 & 180 \\
\hline 20 & $59 / \mathrm{M}$ & 17 & 17 & 16 & 0 & 135 \\
\hline 21 & $62 / \mathrm{F}$ & 17 & 16 & 32 & 13 & 130 \\
\hline 22 & $68 / F$ & 20 & 17 & 16 & 3 & 195 \\
\hline 23 & $54 / \mathrm{M}$ & 23 & 19 & 22 & 10 & 210 \\
\hline
\end{tabular}

These assessments were: (a) unilateral hand tapping count over 30 seconds; $(b)$ time taken to rise from a standard armless chair, walk $6 \mathrm{~m}$, and return to the chair (inability to complete the task within 30 seconds was assigned a time of 30 seconds for the analysis of results); and (c) measurement of regional tremor and dyskinesia according to simple five-point scales $(0=$ none, $1=$ mild, $2=$ moderate, 3 = severe, $4=$ violent/incapacitating) for head, trunk, and each limb (maximum score 24).

Before the levodopa dose ("off" phase) and at the time of peak motor improvement as judged by the serial hand tapping and walking time measurements ("on" phase), patients were scored on a modified Webster disability scale (scoring for 12 areas of motor function giving a maximum disability score of 36$){ }^{3}$ These data were not available in one patient for the initial study.

The amplitude of motor response for each of the motor assessments was the absolute value obtained by subtracting the "on" from "off" results. The duration of response was defined as the time from the onset of response to the return to the baseline motor state. To analyse the changes in motor function with respect to the severity of disease, patients were divided into three subgroups according to disease duration (less than six years, six to 10 years, and greater than 10 years) and also to the modified Webster scale "off" phase disability in the initial study (scores of 9-16, 17-24, and 25-32) as a measure of the severity of motor deficit without pharmacological treatment.

Although no major changes in pharmacological treatment occurred over the study interval, there were some adjustments of both levodopa dose and other anti-parkinsonian medication. The effects of the patients' usual drugs were minimised by the standardised testing conditions described here, but treatment was not otherwise changed before the second assessment on the basis that an alteration of regular drug treatment may have destabilised motor function and introduced additional experimental error.

\section{STATISTICAL ANALYSIS}

Student's $t$ test was used to assess statistical significance. Paired data were analysed in comparing the initial and final study results.

\section{Results}

An unequivocal motor response at the initial assessment of levodopa dose occurred in each patient, accompanied by drug-induced dyskinesia in all but one patient. On the second occasion, 22 patients had a definite response and the remaining patient, although not responding to the standard levodopa $250 \mathrm{mg} /$ carbidopa $25 \mathrm{mg}$ dose, responded to a double dose. This patient's data were included in the assessment of the magnitude and pattern of motor response but were excluded from the analysis of duration and latency.

PROGRESSION OF DISABILITY AND MAGNITUDE OF RESPONSE TO LEVODOPA

The total “off” phase Webster disability score increased over the follow-up period in all but four patients. The mean (range) "off" score was initially $20(9-32)$ and subsequently 25 (17-33) $(p<0.001)$. The increase in "off" score was greatest in those patients with a disease duration of less than six years when initially studied (mean increase $8 \cdot 0$ ) and similar in those with a disease duration of six to 10 years $(3 \cdot 8)$ and more than 10 years $(4 \cdot 2)$. Figure 1 shows these results.

The "on" phase Webster score deteriorated in each patient. The mean (range) initial "on" score was $6 \cdot 2$ (1-13) and the mean (range) final "on" score was 11 (4-22). This deterioration was similar in the three groups classified by duration of disease.

The mean amplitude of levodopa motor response was therefore unchanged over the three year follow-up period. The mean response amplitude increased by 2.0 points in the less than six years' disease duration group 


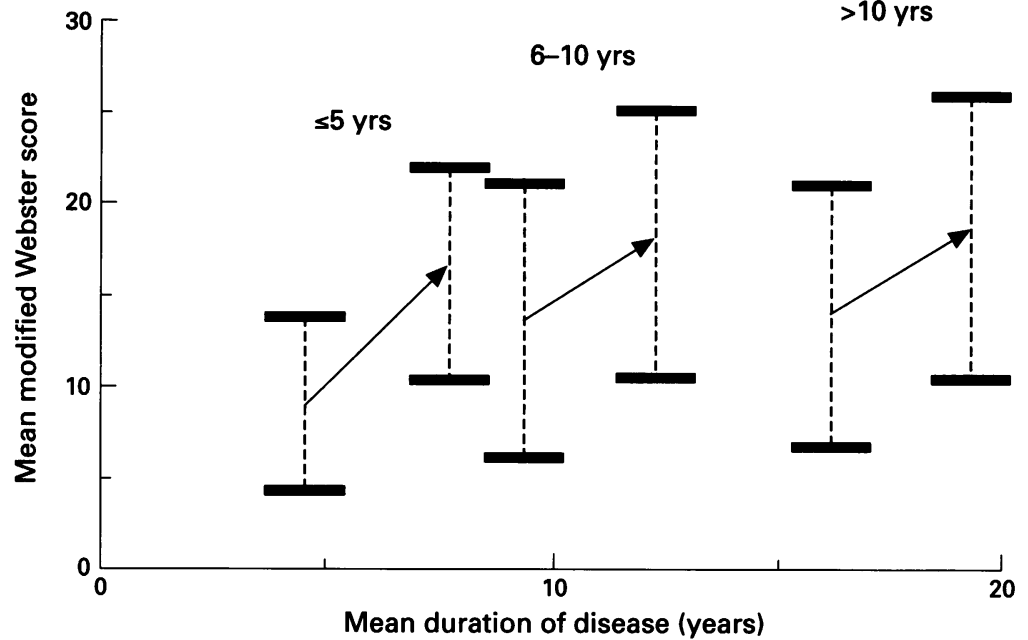

Figure 1 Mean modified Webster scale scores: "on" (upper bars) and "off" (lower bars) at initial and final assessments. Patients are grouped according to the duration of disease at the time of the initial study. but decreased by $0 \cdot 7$ in the six to 10 years' group, and decreased slightly by 0.2 in the more than 10 years' group (differences not significant). Analysis according to initial "off" phase modified Webster score showed that the least disabled patients had a mean amplitude increase of 3.6 over the three years whereas the amplitude in patients with moderate and severe "off" phase disability decreased by $1.6 \quad(p<0.02$ for difference between greatest and least "off” phase disability groups).

The "off" phase walking time increased by three seconds $(p<0.02)$ and the "on" phase time increased by four seconds $(p<0.01)$, but the response amplitude was 12 and 11 in the initial and final studies respectively (no significant difference). Hand tapping counts showed a similar trend with a mild deterioration of the "off" and "on" phase results which were not significant. The response amplitude decreased slightly (23 initial, 21 final).

TOPOGRAPHIC ASPECTS OF MOTOR DISABILITY AND RESPONSE TO LEVODOPA

In the initial study, modified Webster scale scores for arm swing, gait, rigidity, posture,

Table 2 Mean scores in initial and final studies for "off" and "on" phase motor assessments

\begin{tabular}{|c|c|c|c|c|c|c|}
\hline & \multicolumn{3}{|c|}{ Initial study } & \multicolumn{3}{|c|}{ Final study } \\
\hline & "Off" & “On" & Amplitude & "Off" & "On" & Amplitude \\
\hline $\begin{array}{l}\text { Modified Webster sc } \\
\text { Bradykinesia } \\
\text { Rigidity } \\
\text { Posture } \\
\text { Arm swing } \\
\text { Gait } \\
\text { Tremor } \\
\text { Facial movement } \\
\text { Seborrhoea } \\
\text { Speech } \\
\text { Self-care } \\
\text { Balance } \\
\text { Rising from chair } \\
\text { Total }\end{array}$ & $\begin{array}{r}1.9 \\
1.9 \\
1.6 \\
2.5 \\
2.1 \\
1.1 \\
1.5 \\
1.0 \\
1.7 \\
1.9 \\
1.6 \\
1.5 \\
20\end{array}$ & $\begin{array}{l}0.9 \\
0.4 \\
0.3 \\
0.4 \\
0.3 \\
0 \\
0.6 \\
1.0 \\
0.7 \\
0.5 \\
0.8 \\
0.2 \\
6.1\end{array}$ & $\begin{array}{c}1.0 \\
1.5 \\
1.3 \\
2.1 \\
1.8 \\
1.1 \\
0.9 \\
0 \\
1.0 \\
1.4 \\
0.8 \\
1.3 \\
14\end{array}$ & $\begin{array}{r}2 \cdot 6 \\
2 \cdot 2 \\
2 \cdot 1 \\
2 \cdot 8 \\
2 \cdot 8 \\
1 \cdot 1 \\
2 \cdot 0 \\
1 \cdot 4 \\
2 \cdot 0 \\
2.5 \\
2 \cdot 0 \\
1 \cdot 8 \\
25\end{array}$ & $\begin{array}{c}1.1 \\
0.5 \\
1.2 \\
1.0 \\
1.0 \\
0.1 \\
1.1 \\
1.0 \\
1.3 \\
1.1 \\
1.3 \\
0.1 \\
11\end{array}$ & $\begin{array}{r}1.5 \\
1.7 \\
0.9 \\
1.8 \\
1.8 \\
1.0 \\
0.9 \\
0.4 \\
0.7 \\
1.4 \\
0.7 \\
1.7 \\
14\end{array}$ \\
\hline $\begin{array}{l}\text { Hand tapping } \\
\text { Walking time } \\
\text { Dyskinesia } \\
\text { Tremor }\end{array}$ & $\begin{array}{c}29 \\
23 \\
0 \\
3 \cdot 4\end{array}$ & $\begin{array}{l}51 \\
11 \\
9 \cdot 7 \\
0\end{array}$ & $\begin{array}{c}22 \\
12 \\
9 \cdot 7 \\
3 \cdot 4\end{array}$ & $\begin{array}{l}27 \\
26 \\
0 \\
4 \cdot 5\end{array}$ & $\begin{array}{c}47 \\
15 \\
12 \\
0 \cdot 4\end{array}$ & $\begin{array}{c}20 \\
11 \\
12 \\
4 \cdot 1\end{array}$ \\
\hline
\end{tabular}

and self-care contributed most to motor improvement after levodopa doses, whereas seborrhoea, balance, and speech were the least responsive. There was little change in the response pattern over three years, with features reflecting multiple facets of parkinsonian disability such as gait, arm swing, and ability to rise from a chair still contributing to the bulk of improvement, whereas balance, speech, and seborrhoea contributed the least (see table 2).

Modified Webster scale scores representing "midline" motor functions including locomotion (gait, facial movement, speech, balance, rising from chair) were compared with lateralised upper limb motor scores (bradykinesia, rigidity, arm swing, tremor). The amplitude of motor response was conserved for the two sets of scores (mean response amplitude of "midline" scores $1 \cdot 1$ in the initial and final studies, mean "upper limb" score amplitude 1.4 initial and 1.5 final).

DURATION AND LATENCY OF MOTOR RESPONSE There was a $17 \%$ reduction in the mean (range) duration of motor response from 199 (100-345) minutes initially to 168 (43-324) minutes $(p<0.05)$. This reduction was greatest in patients with a disease duration of six to 10 years (mean reduction 61 minutes) and least for those with Parkinson's disease for more than 10 years (mean reduction 16 minutes). Shortening of the response duration over three years varied widely from a reduction to $30 \%$ of the initial response duration to no change or increased duration in the final study in seven patients. Evolution from stable motor function to clinically significant motor oscillation occurred in only one patient who had a response duration of 255 minutes in the two studies. Figure 2 shows the response duration results graphically, grouped according to disease duration and initial "off" phase Webster scale disability.

There was no significant change in the latency to onset of the motor response (mean latency to onset initially 39 minutes and at review 37 minutes).

\section{DYSKINESIA}

The mean dyskinesia score increased by $2 \cdot 3$ $(p<0.01)$ over the three years. Dyskinesia increased most in those with disease duration less than six years (mean change $+4 \cdot 8$ ) compared with those with disease for six to 10 years $(+3 \cdot 6)$ and more than 10 years $(+1 \cdot 0)$.

Sixteen patients had a peak dose response dyskinesia and six others had a biphasic pattern of dyskinesia when first studied. The temporal pattern of dyskinesia remained the same in all but two and the topographic distribution of dyskinesia did not vary. One patient with biphasic dyskinesia and one patient with no dyskinesia initially, had peak dose dyskinesia when restudied. Two patients had prominent "off" phase dystonia in both studies.

\section{TREMOR}

Levodopa caused the complete resolution of tremor in all patients at the initial study and 

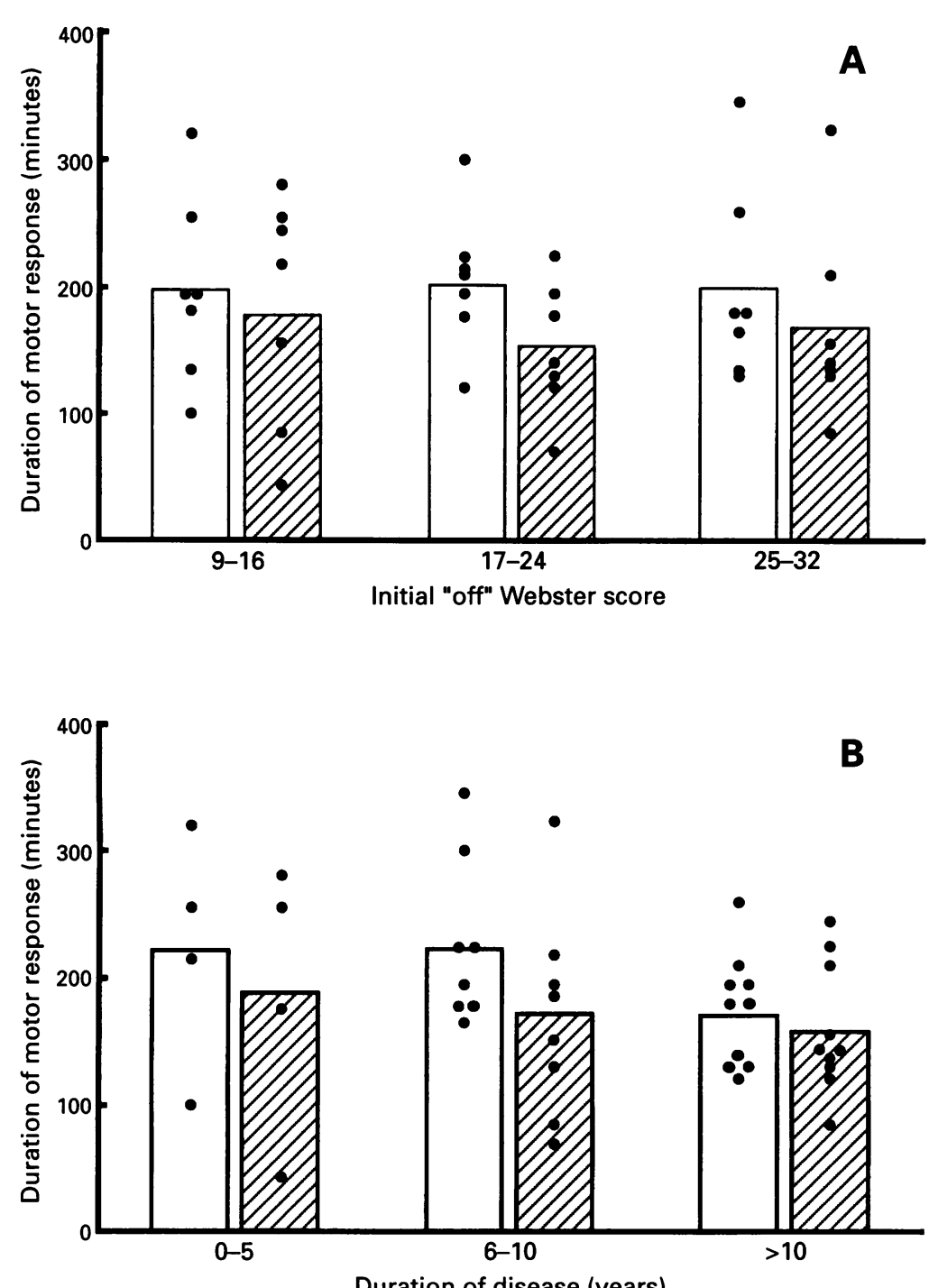

Figure 2 Duration of response to single levodopa/carbidopa dose at initial $(\square)$ and final (ש) studies three years apart. Results divided into groups according to $(A)$ underlying disease severity ("off" modified Webster score) and (B) disease duration.

all but three maintained this response at follow up. The mean tremor score during "off" phases had worsened from 3.4 to 4.5 $(\mathrm{p}<0.01)$.

\section{Discussion}

This unique longitudinal study of motor response characteristics in a cohort of patients with parkinsonian motor fluctuations clarifies several aspects of the deterioration in treatment efficacy due to disease progression. Despite significant increases in motor disability scores and dyskinetic involuntary movements, the capacity to respond to levodopa did not deteriorate significantly, with a roughly equal rate of decrease in "on" and "off" scores and conservation of response amplitude. Semiquantitative disability scale scoring and the less observer-dependent hand tapping and walking measurements showed this trend. If "off" phase motor function can be regarded as a measure of endogenous striatal dopamine deficiency, the progressive reduction in "off" scores reflects progressive nigral cell loss. The motor improvement which occurs during "on" phases depends on the presence of a sufficient number and sensitivity of striatal dopamine receptors plus the integrity of essential non-dopaminergic components of the motor system. Disease progression thus brings increasing reliance on pharmacological striatal dopamine receptor stimulation. This may explain why the best motor state attainable with levodopa treatment decreases and the associated dyskinetic involuntary movements increase. We found no evidence that certain motor deficits such as impairment of speech, gait, and upright postural stability become progressively unresponsive to levodopa as the disease progresses in individual patients, based on the analysis of modified Webster scale scoring and the timed walking task. These aspects of motor function seem to be generally less responsive to levodopa and in some patients who are older at the time of onset of the disease speech and gait are more affected, associated with a modest overall response to levodopa. ${ }^{6}$ In individual patients, however, these deficits seem to retain roughly the same magnitude of response despite worsening "on" and "off" motor function.

The mean age of disease onset in this study group is younger and the mean treatment duration longer than is usual for an unselected parkinsonian population. This reflects our sampling of patients with motor fluctuations, a complication of treatment more common in patients with early onset disease. ${ }^{7}$ The fact, however, that more than $50 \%$ of patients develop motor fluctuations after five years of levodopa treatment ${ }^{8}$ makes these results generally relevant to the management of Parkinson's disease. Our results show significant changes in motor scores over three years which can reasonably be attributed to disease progression. The term "disease progression" as used in this discussion encompasses the clinical manifestation of a variety of pathological changes affecting the motor system, including neuronal loss associated with Lewy bodies in and beyond the substantia nigra as well as the effects of coexistent vascular or Alzheimer-type disease. ${ }^{9}$ Different combinations and rates of progression of these changes may explain some of the variability in the evolution of the pattern of response to levodopa. Our three year follow-up period does not exclude the possibility that some patients with motor fluctuations will eventually show a loss of response amplitude over time, possibly those with more widespread degenerative disease.

The mean duration of the motor response to levodopa decreased with disease progression although this did not occur evenly, with the greatest shortening occurring in patients with relatively short disease duration and little change in patients with longstanding disease. More recently diagnosed patients with more stable motor responses to levodopa had a longer mean motor response duration than those with longstanding disease, many of whom had severe motor fluctuations. The difference in the duration of motor response was not great, however, and there was considerable overlap between the groups. Figure 2 also 
shows that "off" phase disability (taken to reflect the degree of endogenous dopamine deficiency) correlates poorly with response duration. Nutt et al ${ }^{10}$ found that the mean motor response to intravenous levodopa infusion was shorter in patients with a fluctuating response than in those with a stable response and untreated patients, but that, again, considerable overlap was present and the difference did not attain statistical significance. All patients in our study with severe motor fluctuations had large response amplitudes. Although we showed that the mean duration of response to levodopa decreases with disease progression, severe fluctuations were seen in patients with relatively long responses to the test dose, and in one patient motor fluctuations developed over the three year period without any change in the measured duration of response. These observations emphasise the importance of a widening response amplitude and increasing "off" phase disability in the genesis of motor fluctuations.

In many patients the motor response amplitude must increase as a stable motor response early in the course of levodopa treatment gives way to motor fluctuations. This longitudinal study suggests that the amplitude of response is stable with further disease progression and that patients who have a larger response amplitude and develop motor fluctuations may not lose their capacity to benefit from levodopa. This agrees with the clinical experience that when patients with motor fluctuations enter the advanced stages of Parkinson's disease, fluctuation continues with distinct "on" and "off" phases but overall motor func- tion decreases as "off" phase disability becomes extreme, and "on" phases are increasingly marred by involuntary movements and neuropsychiatric toxicity. A patient with motor fluctuations has a response to treatment which is unsatisfactory compared with his or her own responses soon after starting treatment with levodopa or with patients with milder disease. This group of patients appears to maintain a substantial amplitude of response to levodopa over time, however, and may continue to benefit from the manipulation of dopaminergic treatment.

1 Klawans HL, Garvin JS. Treatment of Parkinsonism with L-dopa. Diseases of the Nervous System 1969;30:737-46. Fabbrini G, Juncos J, Mouradian MM, Serati C, Chase Fabbrini G, Juncos J, Mouradian MM, Serati C, Chase
TN. Levodopa pharmacokinetic mechanisms and motor TN. Levodopa pharmacokinetic mechanisms and motor fluctuation

3 Kempster PA, Frankel JP, Bovingdon M, Webster R, Lees AJ, Stern GM. Levodopa peripheral pharmacokinetics and duration of motor response in Parkinson's disease. f Neurol Neurosurg Psychiatry 1989;52:718-23.

4 Bonnet AM, Loria Y, Saint-Hillaire M-H, Lhermitte F, Agid Y. Does long term aggravation of Parkinson's disease result from non-dopaminergic lesions? Neurology 1987;37:1539-42.

5 Rogers D, Lees AJ, Smith E, Trimble M, Stern GM. Bradyphrenia in Parkinson's disease and psychomotor retardation in depressive illness. Brain 1987;110:761-76.

6 Blin J, Dubois B, Bonnet AM, Vidailhet M, Brandabur M, Agid Y. Does ageing aggravate Parkinsonian disability? Agid Y. Does ageing aggravate Parkinsonian
$\mathcal{f}$ Neurol Neurosurg Psychiatry 1991;54:780-2.

7 Hoehn MM. Result of chronic levodopa therapy and its modification by bromocriptine in Parkinson's disease. Acta Neurol Scand 1985;75:97-106.

8 Shaw KM, Lees AJ, Stern GM. The impact of treatment with levodopa in Parkinson's disease. $Q \mathcal{F}$ Med 1980;49: 283-93.

9 Hughes AJ, Daniel SE, Kilford L, Lees AJ. Accuracy of clinical diagnosis of idiopathic Parkinson's disease: a clinico-pathological study of 100 cases. $\mathcal{f}$ Neurol Neurosurg Psychiatry 1992;55:181-4.

10 Nutt JG, Carter JH, Woodward WR, Gancher ST. Pharmacodynamics of chronic L-dopa administration. Neurology 1990;40(suppl):292. 